\title{
Efficient frequency-transient co-simulation of coupled heat-electromagnetic problems
}

\author{
Christof Kaufmann ${ }^{1 *}$, Michael Günther ${ }^{2}$, Daniel Klagges ${ }^{3}$, Michael Knorrenschild ${ }^{1}$, Matthias Richwin ${ }^{3}$, \\ Sebastian Schöps ${ }^{4}$ and E Jan W ter Maten ${ }^{2,5}$
}

\section{"Correspondence:}

christof.kaufmann@hs-bochum.de ${ }^{1}$ Fachbereich Elektrotechnik und Informatik, Hochschule Bochum, Bochum, 44801, Germany Full list of author information is available at the end of the article

\begin{abstract}
Background: With the recent advent of inductive charging systems all major automotive manufacturers develop concepts to wirelessly charge electric vehicles. Efficient designs require virtual prototyping that accounts for electromagnetic and thermal fields. The coupled simulations can be computationally very costly. This is because of the high frequencies in the electromagnetic part. This paper derives a mixed frequency-transient model as approximation to the original problem. We propose a co-simulation such that the electromagnetic part is simulated in the frequency domain while the thermal part remains in time domain.

Results: The iteration scheme for the frequency-transient model is convergent for high frequency excitation. The error bound improves quadratically with increasing frequency.

Conclusions: The frequency-transient model is very efficient for coupled heat-electromagnetic simulations since the time scales typically differ by several orders of magnitude. The time steps of the full system can be chosen according to the heat subsystem only.
\end{abstract}

MSC: $35 \mathrm{~K} 05 ; 35 \mathrm{Q} 61 ; 65 \mathrm{Z05} ; 78 \mathrm{~A} 25 ; 78 \mathrm{M} 12 ; 80 \mathrm{M} 25$

Keywords: inductive charging; coupled simulation; co-simulation; electromagnetic; heat; modelling; dynamic iteration

\section{Introduction}

With the recent advent of inductive power charging systems and wireless power transmission in consumer and mobile phone technology, [1], all major automotive manufacturers develop concepts to wirelessly charge electric vehicles, both plug-in and pure electric vehicles (EV). For example the prototype from the Leopold Kostal GmbH \& Co. KG of such an inductive charging station is shown in Figure 1. The necessity to charge EVs with today's battery technology after every prolonged use - at least every night - is seen as one of the major drawbacks in the usability of EVs. A system to automate the charging process would reduce the burden on the driver; it could increase the acceptance of EVs, and, in the case of plug-in hybrid EVs, it could help to further reduce the $\mathrm{CO}_{2}$ footprint since the battery of the plug-in hybrid could always be considered fully charged. This is important for the calculation of the fleet $\mathrm{CO}_{2}$ emission.

A future inductive charging system does not necessarily exhibit a lower efficiency than a comparable conductive charging system, since there are only a few additional compo-

\section{Springer}

(c) 2014 Kaufmann et al.; licensee Springer. This is an Open Access article distributed under the terms of the Creative Commons Attribution License (http://creativecommons.org/licenses/by/2.0), which permits unrestricted use, distribution, and reproduction in any medium, provided the original work is properly cited. 


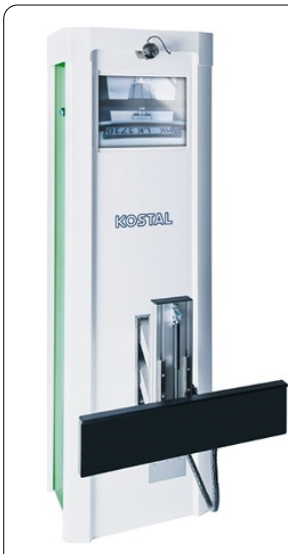

(a) Charge station.

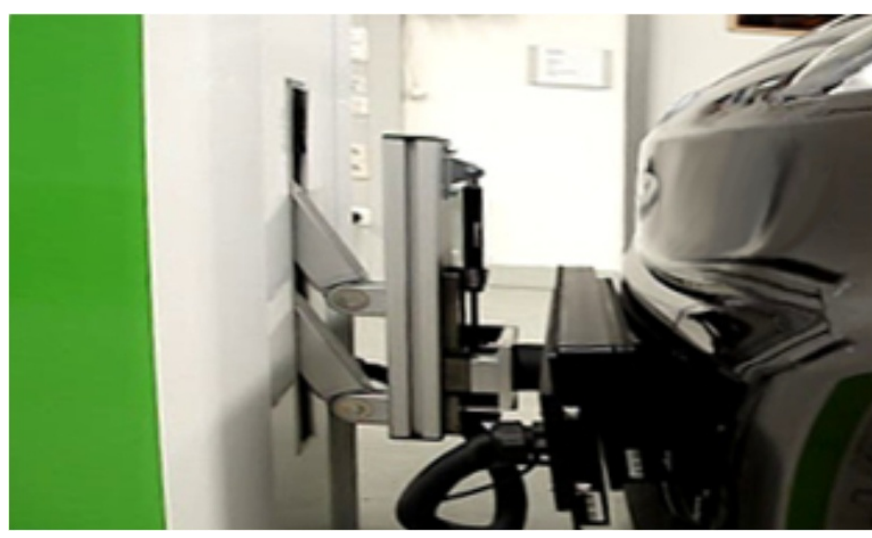

(b) Charging of a electric vehicle.

Figure 1 Prototype of an inductive charging station that charges the vehicle through its number plate (Images from Leopold Kostal GmbH \& Co. KG).

nents; in a simplified view, the inductive charging system could be considered as a conductive charging system that has been cut in half in the middle of the transformer. There are, however, certain aspects that require attention and detailed design and optimization. These include positioning tolerances of the stationary ('primary') and car-mounted ('secondary') coils, magnetic stray fields, and thermal aspects.

The efficiency of both conductive and inductive charging systems is aimed well above $90 \%$, measured from the primary AC connection to the drive train battery. But even with this high efficiency, at $3.5 \mathrm{~kW}$ of first generation systems charging power there is a nonnegligible amount of heat to be dissipated. Later generations with even higher power will further increase the heat load on the components. This heat load is the result of several different processes, namely resistance losses due to DC resistance and proximity effects, ferrite core losses and switching losses in the active semiconductor switching components. The main effects appear at the same frequency range as the magnetic field, which is of the order of 10-100 kHz. The resulting temperature however changes on much slower timescales, in the order of minutes, determined by the heat capacity and the (relatively large) mass of the involved components. This electromagnetic-thermal problem is fully coupled, as many of the material parameters show a significant thermal dependence. Typical ferrite core losses, for instance, are minimal at temperatures around $90^{\circ} \mathrm{C}$ and increase below and above this temperature. This drives the equilibrium temperature of the ferrite material always close to this temperature, if the dissipated power is small enough, or makes the system thermally unstable, if the heat power is too high.

Engineering samples of such systems are expensive, heavy, possibly dangerous to operate, and take a lot of time to build and optimize. Virtual prototyping using efficient simulation methods accelerates this process. There are different methodologies and models available, [2].

The paper is structured as follows: In Section 2, we propose a particular frequencytransient model for electromagnetic-thermal problems. The electromagnetic (EM) field is considered at high frequencies, where the time scale of the heat conduction is significantly lower than the time scale of the EM field. This can be exploited in the modeling. We propose a co-simulation scheme, similar to dynamic iteration [3], and analyze its con- 
vergence properties in Section 3. The analysis exhibits interesting results, especially for high frequencies. Possibilities to generalize this model are also discussed. Section 4 validates the results by a numerical simulation of a simplified model of the inductive charging system shown in Figure 1.

In contrast to [2], where the different ways of co-simulation are discussed, we focus here on modeling and analysis of the frequency-transient model.

\section{Modeling}

In this section, we derive a model, which describes the electromagnetic field coupled to the temperature in the materials. For that, in Section 2.1 Maxwell's equations are introduced with temperature dependent material parameters. The conduction of the heat is described in Section 2.2 by the heat equation together with an electromagnetic power term as source to describe the Joule losses of the EM field. Finally, in Section 2.3 assumptions and approximations lead to the frequency-transient model [4].

\subsection{Maxwell's equations}

Maxwell's equations read

$$
\begin{aligned}
& \nabla \cdot \mathbf{D}=\rho, \\
& \nabla \cdot \mathbf{B}=0, \\
& \nabla \times \mathbf{E}=-\frac{\partial \mathbf{B}}{\partial t}, \\
& \nabla \times \mathbf{H}=\frac{\partial \mathbf{D}}{\partial t}+\mathbf{J},
\end{aligned}
$$

where $\mathbf{E}$ and $\mathbf{H}$ are the electric and magnetic field strength, $\mathbf{D}$ and $\mathbf{B}$ are the electric and magnetic flux densities, $\rho$ and $\mathbf{J}$ are the electric charge and current densities. These laws are supported by the constitutive relations

$$
\mathbf{D}=\varepsilon \mathbf{E}, \quad \mathbf{B}=\mu \mathbf{H} \quad \text { and } \quad \mathbf{J}=\sigma(T) \mathbf{E}+\mathbf{J}_{\text {src }},
$$

where the permittivity $\varepsilon$ and permeability $\mu$ parameters depend only on space while the conductivity $\sigma$ may also depend on the temperature $T$. In this way the EM field solution is a function of the temperature distribution (parameter coupling). However, the source current density $\mathbf{J}_{\mathrm{src}}$ describes an external excitation $[4,5]$; it is assumed to be independent of the temperature. Now to reduce the unknowns in Maxwell's equations, we introduce the magnetic vector potential $\mathbf{A}$ and the electric scalar potential $\varphi$ as

$$
\mathbf{E}=-\nabla \varphi-\frac{\partial \mathbf{A}}{\partial t} \quad \text { with } \mathbf{B}=\nabla \times \mathbf{A} .
$$

By using these potentials, (2) and (3) are fulfilled automatically. From (4) we get

$$
\nabla \times\left(\mu^{-1} \nabla \times \mathbf{A}\right)+\varepsilon \frac{\partial^{2} \mathbf{A}}{\partial t^{2}}+\sigma(T) \frac{\partial \mathbf{A}}{\partial t}+\varepsilon \nabla \frac{\partial \varphi}{\partial t}+\sigma(T) \nabla \varphi=\mathbf{J}_{\mathrm{src}} .
$$

With Buchholz gauge transformation $(\nabla \varphi=0)$ this reduces to

$$
\nabla \times\left(\mu^{-1} \nabla \times \mathbf{A}\right)+\varepsilon \frac{\partial^{2} \mathbf{A}}{\partial t^{2}}+\sigma(T) \frac{\partial \mathbf{A}}{\partial t}=\mathbf{J}_{\text {src }}
$$


The so-called curl-curl equation will be treated in the following on a finite domain with adequate boundary conditions (BC) and initial values (IV).

For the low frequency range, where inductive effects dominate, usually the displacement current $\partial \mathbf{D} / \partial t \hat{=} \omega^{2} \varepsilon \hat{\mathbf{A}}$ can be disregarded. This is called magnetoquasistatic formulation. For details on these formulations we refer to [6]. Here we are interested in the high frequency range. Therefore our model is based on the full Maxwell formulation (1)-(4).

\subsection{Heat equation}

The classical heat equation describes conduction of heat in materials:

$$
\rho c \frac{\partial T}{\partial t}=\nabla \cdot(k \nabla T)+Q
$$

with heat conductivity $k$, density $\rho$ and specific heat capacity $c$, all constant in time and again on a finite domain with $\mathrm{BC}$ and IV. The term $Q$ represents the Joule losses of the EM field. Hence we use a source coupling to connect the heat equation with the EM curlcurl equation (5). For simplicity we consider only eddy-current and Joule losses and thus obtain

$$
Q(\mathbf{A}, T)=\mathbf{J} \cdot \mathbf{E}=\sigma(T) \mathbf{E} \cdot \mathbf{E}+\mathbf{J}_{\mathrm{src}} \cdot \mathbf{E}=\sigma(T) \frac{\partial \mathbf{A}}{\partial t} \cdot \frac{\partial \mathbf{A}}{\partial t}-\mathbf{J}_{\mathrm{src}} \cdot \frac{\partial \mathbf{A}}{\partial t} .
$$

The power term is further simplified in the next section.

\subsection{Frequency-transient model}

Now we aim at a model which allows an efficient simulation. The model consisting of (5) and (6) with (7) is defined in the time domain. A multirate co-simulation scheme could simulate both equations with different time steps. However, for a fast varying source current density the main part of the computational costs is caused by the simulation of (5). A discussion of single-rate and multirate schemes can be found in [2]. We will reduce these costs further by refining the model.

Since the temperature is only slowly varying in comparison to the EM field in (5) the temperature $T$, where $\sigma$ is evaluated at, can be averaged by

$$
\tilde{T}_{i}:=\frac{1}{\tau_{i+1}-\tau_{i}} \int_{\tau_{i}}^{\tau_{i+1}} T(t) d t
$$

Thus we use $\sigma(T(t)) \approx \sigma\left(\tilde{T}_{i}\right)$ for $t \in\left[\tau_{i}, \tau_{i+1}\right]$ and then (5) can be approximated by

$$
\nabla \times\left(\mu^{-1} \nabla \times \mathbf{A}\right)+\varepsilon \frac{\partial^{2} \mathbf{A}}{\partial t^{2}}+\sigma\left(\tilde{T}_{i}\right) \frac{\partial \mathbf{A}}{\partial t}=\mathbf{J}_{\mathrm{src}}
$$

However, this is still in time domain. To allow a solution in frequency domain, we assume a time harmonic source current density

$$
\mathbf{J}_{\mathrm{src}}=\frac{1}{2} \hat{\mathbf{J}}_{\mathrm{src}} e^{j \omega t}+\frac{1}{2} \overline{\hat{\mathbf{J}}_{\mathrm{sc}}} e^{-j \omega t}
$$

where $\hat{\mathbf{J}}_{\text {src }}$ is a complex phasor. It follows for $\mu$ and $\varepsilon$ independent of $\mathbf{A}$ that

$$
\mathbf{A}(t)=\frac{1}{2}\left(\hat{\mathbf{A}}_{c}\left(\tilde{T}_{i}\right) e^{j \omega t}+\overline{\hat{\mathbf{A}}_{c}\left(\tilde{T}_{i}\right)} e^{-j \omega t}\right),
$$


where the complex phasor $\hat{\mathbf{A}}_{c}$ is the solution for $\hat{\mathbf{J}}_{\text {src }}$. This means the amplitude is constant within the time interval $\left[\tau_{i}, \tau_{i+1}\right]$.

Let us look at (7) again, but now with the approximation $\sigma(T(t)) \approx \sigma\left(\tilde{T}_{i}\right)$. The dot product is the usual real inner product.

$$
\begin{aligned}
Q\left(\mathbf{A}, \tilde{T}_{i}\right)= & \sigma\left(\tilde{T}_{i}\right) \frac{\partial \mathbf{A}}{\partial t} \cdot \frac{\partial \mathbf{A}}{\partial t}-\mathbf{J}_{\mathrm{src}} \cdot \frac{\partial \mathbf{A}}{\partial t} \\
= & -\sigma\left(\tilde{T}_{i}\right) \frac{\omega^{2}}{4}\left(\hat{\mathbf{A}}_{c} \cdot \hat{\mathbf{A}}_{c} e^{j 2 \omega t}-2 \hat{\mathbf{A}}_{c} \cdot \overline{\hat{\mathbf{A}}_{c}}+\overline{\hat{\mathbf{A}}_{c}} \cdot \overline{\hat{\mathbf{A}}_{c}} e^{-j 2 \omega t}\right) \\
& -j \frac{\omega}{4}\left(\hat{\mathbf{J}}_{\mathrm{src}} \cdot \hat{\mathbf{A}}_{c} e^{j 2 \omega t}-\hat{\mathbf{J}}_{\mathrm{src}} \cdot \overline{\hat{\mathbf{A}}_{c}}+\overline{\hat{\mathbf{J}}_{\mathrm{src}}} \cdot \hat{\mathbf{A}}_{c}-\overline{\hat{\mathbf{J}}_{\mathrm{src}}} \cdot \overline{\hat{\mathbf{A}}_{c}} e^{-j 2 \omega t}\right),
\end{aligned}
$$

where $\hat{\mathbf{A}}_{c}=\hat{\mathbf{A}}_{c}\left(\tilde{T}_{i}\right)$. Now we are interested in the mean power loss in the time interval $\left[\tau_{i}, \tau_{i+1}\right]:$

$$
\tilde{Q}_{i}:=\frac{1}{\tau_{i+1}-\tau_{i}} \int_{\tau_{i}}^{\tau_{i+1}} Q\left(\mathbf{A}(t), \tilde{T}_{i}\right) d t
$$

and thus all parts with $e^{ \pm j 2 \omega t}$ vanish (for interval length being a multiple of the half period length, i.e. $\tau_{i+1}-\tau_{i}=c \frac{\pi}{\omega}$, where $c \in \mathbb{N}$ ). Then we are left with

$$
\tilde{Q}_{i}=\sigma\left(\tilde{T}_{i}\right) \frac{\omega^{2}}{2}\left\|\hat{\mathbf{A}}_{c}\left(\tilde{T}_{i}\right)\right\|_{c}^{2}+\frac{\omega}{2} \operatorname{Im}\left(\hat{\mathbf{J}}_{\mathrm{src}} \cdot \overline{\hat{\mathbf{A}}_{c}\left(\tilde{T}_{i}\right)}\right)
$$

where $\|\mathbf{A}\|_{c}:=\mathbf{A} \cdot \overline{\mathbf{A}}$ is the Euclidean norm for complex vectors $\mathbf{A}$. Now the simplified curl-curl equation (8) can be considered in frequency domain (with vector potential $\hat{\mathbf{A}}_{c}=$ $\left.\hat{\mathbf{A}}_{c}\left(\tilde{T}_{i}\right)\right)$ along with the simplified heat equation (14) left in time domain:

$$
\begin{aligned}
& \left(j \omega \sigma\left(\tilde{T}_{i}\right)-\omega^{2} \varepsilon\right) \hat{\mathbf{A}}_{c}+\nabla \times\left(\mu^{-1} \nabla \times \hat{\mathbf{A}}_{c}\right)=\hat{\mathbf{J}}_{\mathrm{src}}, \\
& \rho c \frac{\partial T}{\partial t}=\nabla \cdot(k \nabla T)+\tilde{Q}_{i} .
\end{aligned}
$$

The curl-curl equation is formulated with constant material parameters in frequency domain. Thus, only a linear, complex system has to be solved once for each time window, instead for each time step of the curl-curl equation in time domain.

\section{Algorithm}

We will now discuss the algorithm to simulate heat-EM problems with the frequencytransient model. After discretization, the model is solved in a Gauss-Seidel scheme, which can be interpreted as co-simulation. It is comparable with a dynamic iteration for time integration. Section 3.1 will briefly discuss the co-simulation scheme. In Section 3.2 the convergence analysis for the iteration is proved.

\subsection{Method}

The co-simulation scheme uses (13) and (14) in a discretized form. For simplicity we assume Finite Integration Technique (FIT) [7-9] for spatial discretization and then for time discretization the implicit Euler method. As an alternative to FIT discretization, one could use the (lowest order) Finite Element or Boundary Element Methods [10, 11] with the 
drawback of non-diagonal material matrices. This would complicate the following derivations. However, in FIT-notation the curl-curl equation (13) becomes

$$
\left(j \omega \mathbf{M}_{\sigma}^{l}-\omega^{2} \mathbf{M}_{\varepsilon}+\mathbf{C}^{\top} \mathbf{M}_{\nu} \mathbf{C}\right) \widehat{\mathbf{a}}^{l+1}=\widehat{\mathbf{j}}_{\mathrm{s}}
$$

with (diagonal) positive semi-definite matrix for electric conductivity $\mathbf{M}_{\sigma}$, (diagonal) positive definite matrices for permittivity and reluctivity $\mathbf{M}_{\epsilon}, \mathbf{M}_{v}$, discrete curl operators $\mathbf{C}$ and $\mathbf{C}^{\top}$. Note, that $\mathbf{M}_{\sigma}^{l}:=\mathbf{M}_{\sigma}\left(\mathbf{T}^{l}\right)$, where $\mathbf{T}^{l}$ denotes the discretized temperature at the $l$ th iteration step. Let $n$ denote the number of nodes in the computational domain, then the matrices are from $\mathbb{R}^{3 n \times 3 n}$; the discretized (facet-integrated) source current density $\widehat{\mathbf{j}}_{\mathrm{s}} \in \mathbb{R}^{3 n}$ and the (edge-integrated) magnetic vector potential $\widehat{\mathbf{a}} \in \mathbb{R}^{3 n}$ are ordered in $x y z$ direction, e.g., $\widehat{\mathbf{a}}^{\top}:=\left[\widehat{\mathbf{a}}_{x}^{\top}, \widehat{\mathbf{a}}_{y}^{\top}, \widehat{\mathbf{a}}_{z}^{\top}\right]$, [9]. For the heat equation, (14) gives

$$
\left(\mathbf{M}_{\rho c}+h_{i} \tilde{\mathbf{S}} \mathbf{M}_{k} \tilde{\mathbf{S}}^{\top}\right) \mathbf{T}^{l+1}=\mathbf{M}_{\rho c} \mathbf{T}_{i}+h_{i} \frac{\omega}{2} \mathbf{P}\left[\omega \mathbf{M}_{\sigma}^{l+1} \widehat{\mathbf{a}}^{l+1} \circ \overline{\mathbf{a}^{l+1}}+\operatorname{Im}\left(\widehat{\hat{\mathbf{j}}_{\mathrm{s}}} \circ \overline{\widehat{\mathbf{a}}^{l+1}}\right)\right]
$$

where $\circ$ denotes the Hadamard (element wise) product, diagonal positive definite matrices for thermal conductivity and volumetric heat capacity $\mathbf{M}_{k}, \mathbf{M}_{\rho c}$, discrete divergence and gradient operators $\tilde{\mathbf{S}},-\tilde{\mathbf{S}}^{\top}$ on the dual grid, respectively. The matrix $\mathbf{P} \in \mathbb{R}^{n \times 3 n}$ averages and sums up the discrete losses, cf. (12). We use $\mathbf{P}:=[\mathbf{I}, \mathbf{I}, \mathbf{I}]$ with $\mathbf{I} \in \mathbb{R}^{n \times n}$ being the identity matrix. However other choices are possible and in [12] a more sophisticated averaging is proposed for $\mathbf{P}$.

To simulate this model, (15) and (16) are solved successively. This can be repeated for one time step until convergence (see Algorithm 1), similarly as done in Gauss-Seidel schemes. Here, we call it co-simulation. The scheme is also depicted in Figure 2. However, since convergence cannot be guaranteed for such schemes in general, a proof is given in Section 3.2.

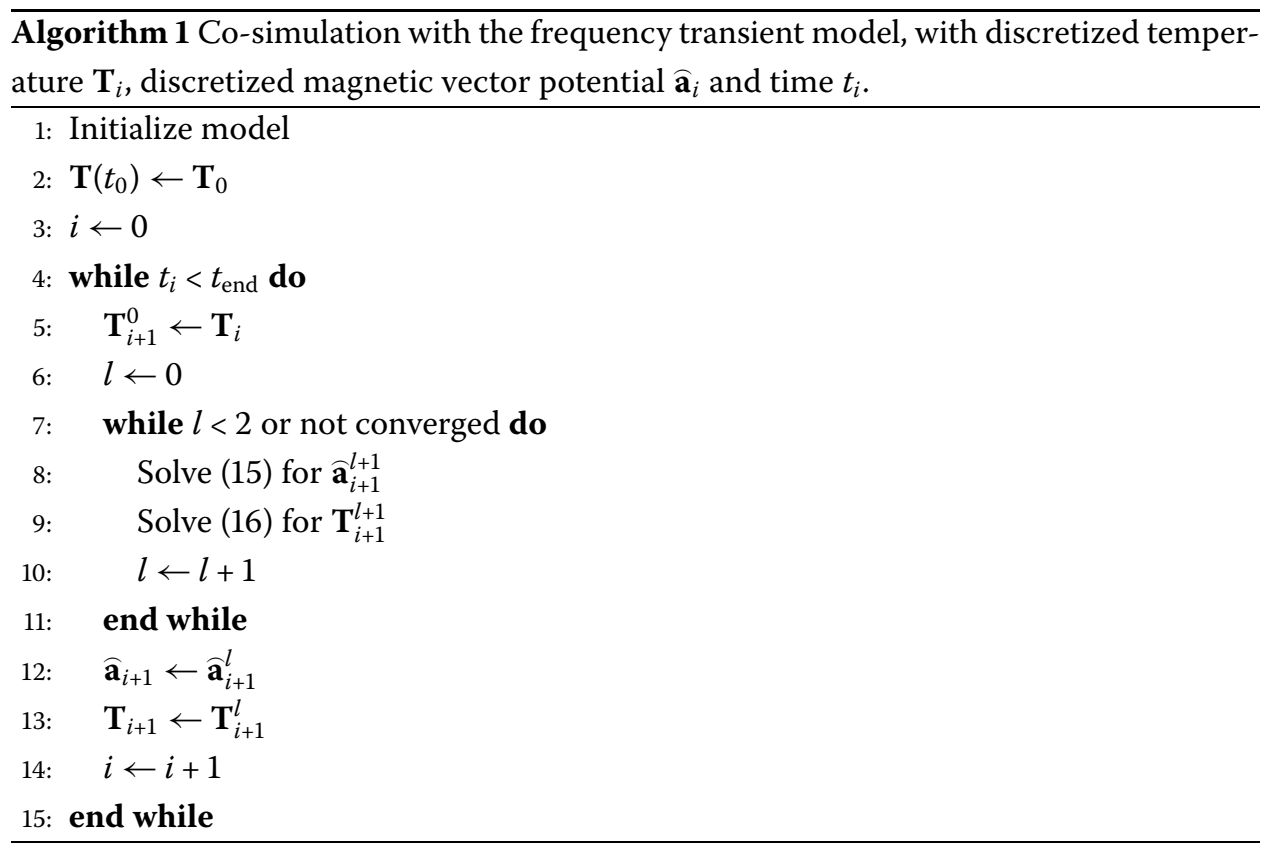




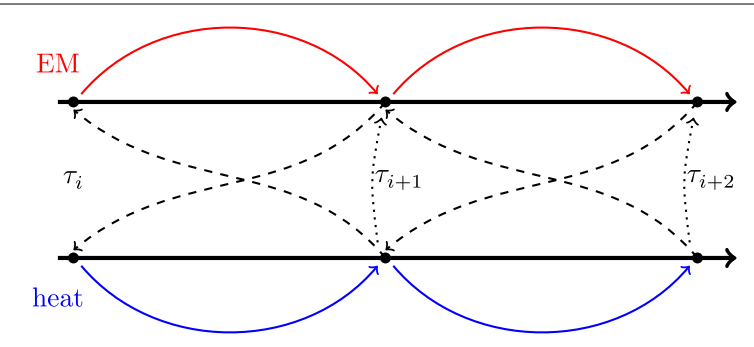

Figure 2 Frequency-transient co-simulation approach.

From Algorithm 1 it can be seen that time steps are chosen according to the time constant of the heat equation and only one (complex) linear system is solved per iteration for the curl-curl equation in frequency domain. Thus the time step size is independent of the excitation frequency $\omega$. In contrast, a time domain solution would require many time steps per period $2 \pi / \omega$ and consequently the solution of a large number of real-valued linear systems. This is the reason, why the model is very efficient for high frequencies since there is multirate behavior.

\subsection{Convergence analysis}

We first verify that the Maxwell operator is bounded:

Lemma Let the EM problem (15) be given with adequate $B C, I V$ for a frequency $\omega>\omega_{0}$. Then the inverse of the discrete Maxwell operator exists and is bounded

$$
\left\|\left(j \omega \mathbf{M}_{\sigma}(\mathbf{T})-\omega^{2} \mathbf{M}_{\varepsilon}+\mathbf{C}^{\top} \mathbf{M}_{\nu} \mathbf{C}\right)^{-1}\right\| \leq C_{0}(\omega)
$$

for some frequency dependent upper bound $C_{0}(\omega)$ that is independent of the temperature.

Proof Beforehand we introduce some abbreviations:

$$
\mathbf{X}:=\omega^{2} \mathbf{M}_{\varepsilon}-\mathbf{C}^{\top} \mathbf{M}_{\nu} \mathbf{C}, \quad \mathbf{Z}:=-\mathbf{X}+j \omega \mathbf{M}_{\sigma}
$$

For $\omega>\omega_{0}$ for some $\omega_{0}$ the matrix $\mathbf{X}=\mathbf{X}(\omega)$ is real, symmetric positive definite and $\mathbf{M}_{\sigma}=\mathbf{M}_{\sigma}(\mathbf{T})$ is real, diagonal, positive semi-definite. Then

$$
\mathbf{Z}=\left(-\mathbf{X}+j \omega \mathbf{M}_{\sigma}\right)=\mathbf{X}^{1 / 2}\left(-\mathbf{I}+j \omega \mathbf{X}^{-1 / 2} \mathbf{M}_{\sigma} \mathbf{X}^{-1 / 2}\right) \mathbf{X}^{1 / 2},
$$

where $\mathbf{X}^{1 / 2}:=\mathbf{U} \boldsymbol{\Sigma}^{1 / 2} \mathbf{U}^{-1}$ for an eigendecomposition $\mathbf{X}=\mathbf{U} \boldsymbol{\Sigma} \mathbf{U}^{-1}$.

Now let $\mathbf{A}:=\mathbf{X}^{-1 / 2} \mathbf{M}_{\sigma} \mathbf{X}^{-1 / 2}$, which is real and symmetric positive semi-definite. It follows that the eigenvalue decomposition is $\mathbf{A}=\mathbf{Q}^{-1} \mathbf{\Lambda} \mathbf{Q}$, with $\|\mathbf{Q}\|=1$. Then

$$
\begin{aligned}
\mathbf{Z}^{-1} & =\mathbf{X}^{-1 / 2}(-\mathbf{I}+j \omega \mathbf{A})^{-1} \mathbf{X}^{-1 / 2} \\
& =\mathbf{X}^{-1 / 2}\left(-\mathbf{Q}^{-1} \mathbf{Q}+j \omega \mathbf{Q}^{-1} \boldsymbol{\Lambda} \mathbf{Q}\right)^{-1} \mathbf{X}^{-1 / 2} \\
& =\mathbf{X}^{-1 / 2} \mathbf{Q}^{-1}(-\mathbf{I}+j \omega \mathbf{\Lambda})^{-1} \mathbf{Q} \mathbf{X}^{-1 / 2},
\end{aligned}
$$




$$
\begin{aligned}
\left\|\mathbf{Z}^{-1}\right\| & \leq\left\|\mathbf{X}^{-1 / 2}\right\|\left\|\mathbf{Q}^{-1}\right\|\left\|(-\mathbf{I}+j \omega \mathbf{\Lambda})^{-1}\right\|\|\mathbf{Q}\|\left\|\mathbf{X}^{-1 / 2}\right\| \\
& \leq\left\|\mathbf{X}^{-1 / 2}\right\| \cdot 1 \cdot \frac{1}{\sqrt{1+\omega^{2} \lambda_{\min }^{2}}} \cdot 1 \cdot\left\|\mathbf{X}^{-1 / 2}\right\|=\left\|\mathbf{X}^{-1}\right\|=: C_{0}(\omega)
\end{aligned}
$$

because $\mathbf{A}$ is semidefinite.

The Lemma guarantees solvability of Maxwell's equations independent of the temperature. However, generalizations are possible but we focus here on the high frequency case because it exhibits a distinct multirate potential. In practice the time-harmonic approach can be used over a wide range of possible excitation frequencies. In particular for lowfrequencies where the displacement currents are often disregarded [13, 14]. Furthermore for other choices of gauging similar results are found. Also in pure transient simulation [15], a wide range of excitation frequencies is covered in practice.

Theorem Let the coupled problem (15)-(16) be given with adequate BC, IV for a frequency $\omega>\omega_{0}$. We assume for nonlinear materials, e.g., metals, positivity and differentiability for $\sigma(T)$ w.r.t. temperature $T$ and $\sigma^{\prime}<0$. Let the exact (monolithic) solution be denoted by $\mathbf{a}^{\infty}$ and $\mathbf{T}^{\infty}$, then the iteration is convergent for $h_{i}$ small enough with

$$
\left\|\mathbf{T}^{l+1}-\mathbf{T}^{\infty}\right\| \leq C(\omega) h_{i}\left\|\mathbf{T}^{l}-\mathbf{T}^{\infty}\right\|
$$

where $C(\omega)$ is uniformly bounded. Furthermore, we have $C(\omega)=\mathcal{O}\left(\frac{1}{\omega^{2}}\right)$ for sufficiently large $\omega$.

Proof Consider the inner loop of Algorithm 1 (steps 8 and 9). It consists of (15) and (16). We prove convergence for this inner loop and use the same abbreviations as introduced in (18) with the shortcut $\mathbf{Z}^{l}:=\mathbf{Z}\left(\mathbf{T}^{l}\right)$. Then we deduce from (15)

$$
\mathbf{Z}^{l} \mathbf{a}^{l+1}=\widehat{\hat{\mathbf{j}}}_{\mathrm{s}} \quad \Leftrightarrow \quad \widehat{\mathbf{a}}^{l+1}=\left(\mathbf{Z}^{l}\right)^{-1} \widehat{\hat{\mathbf{j}}}_{\mathrm{s}}
$$

due to the Lemma above. We define $\mathbf{N}:=\tilde{\mathbf{S}} \mathbf{M}_{k} \tilde{\mathbf{S}}^{\top}$, use (16) with $\mathbf{T}^{\infty}$ and $\widehat{\mathbf{a}}^{\infty}$ subtract it from (16)

$$
\begin{aligned}
\left(\mathbf{M}_{\rho c}+h_{i} \mathbf{N}\right)\left(\mathbf{T}^{l+1}-\mathbf{T}^{\infty}\right)= & h_{i} \frac{\omega^{2}}{2} \mathbf{P}\left(\mathbf{M}_{\sigma}^{l+1} \widehat{\mathbf{a}}^{l+1} \circ \overline{\mathbf{\mathbf { a }}^{l+1}}-\mathbf{M}_{\sigma}^{\infty} \widehat{\mathbf{a}}^{\infty} \circ \overline{\widehat{\mathbf{a}}^{\infty}}\right) \\
& +h_{i} \frac{\omega}{2} \mathbf{P} \operatorname{Im}\left(\widehat{\hat{\mathbf{j}}_{\mathrm{s}}} \circ \overline{\left(\overline{\mathbf{a}}^{l+1}-\widehat{\mathbf{a}}^{\infty}\right)}\right) .
\end{aligned}
$$

Next, by adding and subtracting $h_{i} \frac{\omega^{2}}{2} \mathbf{P} \mathbf{M}_{\sigma}^{\infty} \widehat{\mathbf{a}}^{l+1} \circ \overline{\widehat{\mathbf{a}}^{l+1}}$, we have

$$
\begin{aligned}
\left(\mathbf{M}_{\rho c}\right. & \left.+h_{i} \mathbf{N}\right)\left(\mathbf{T}^{l+1}-\mathbf{T}^{\infty}\right) \\
= & h_{i} \frac{\omega^{2}}{2} \mathbf{P}\left(\mathbf{M}_{\sigma}^{l+1}-\mathbf{M}_{\sigma}^{\infty}\right)\left(\widehat{\mathbf{a}}^{l+1} \circ \overline{\mathbf{a}^{l+1}}\right) \\
& +h_{i} \frac{\omega^{2}}{2} \mathbf{P M}_{\sigma}^{\infty}\left(\widehat{\mathbf{a}}^{l+1} \circ \overline{\mathbf{a}^{l+1}}-\widehat{\mathbf{a}}^{\infty} \circ \overline{\widehat{\mathbf{a}}^{\infty}}\right)+h_{i} \frac{\omega}{2} \mathbf{P} \operatorname{Im}\left(\widehat{\hat{\mathbf{j}}}_{\mathrm{s}} \circ \overline{\left(\widehat{\mathbf{a}}^{l+1}-\widehat{\mathbf{a}}^{\infty}\right)}\right) .
\end{aligned}
$$


Figure 3 Mimetic discretizations of Maxwell's equations (e.g. by FIT) use a primal and dual grid. The discrete temperatures $T_{k}$ are located at primary nodes, the line-integrated electric field and the magnetic vector potentials $\widehat{e}_{k}=\widehat{a}_{k}^{\prime}$ are defined at primary edges and finally the facet-integrated source current density $\widehat{\hat{j}}_{k}$ at dual surfaces. The material laws relate the quantities on both grids, e.g., the conductivity connects by Ohm's Law the currents and voltages, i.e. $\widehat{\widehat{j}}_{k}=\sigma_{k}\left(T_{k}\right) \widehat{e}_{k}$. The figure shows the case for a current in $x$-direction.

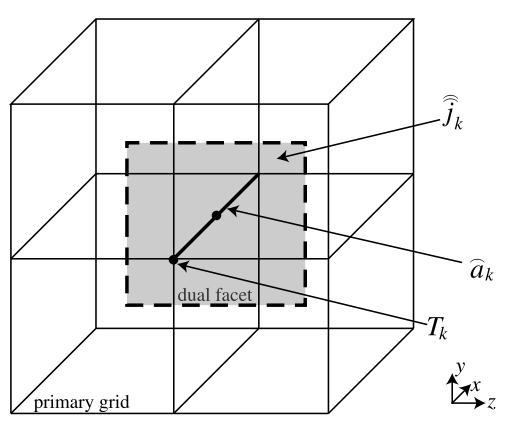

Now, let us consider the term

$$
\left(\mathbf{M}_{\sigma}^{l+1}-\mathbf{M}_{\sigma}^{\infty}\right)\left(\widehat{\mathbf{a}}^{l+1} \circ \overline{\mathbf{a}^{l+1}}\right) .
$$

The conductivity matrix $\mathbf{M}_{\sigma}$ is a diagonal matrix for any iteration step. We assume, that the $k$ th component $\sigma_{k}(1 \leq k \leq 3 n)$ depends only on the neighboring temperature $T_{k}:=$ $\left(\mathbf{P}^{\top} \mathbf{T}\right)_{k}$, see Figure 3 . Thus the $k$ th component of the term can be written as

$$
\widehat{a}_{k}^{l+1}\left(\sigma_{k}\left(T_{k}^{l+1}\right)-\sigma_{k}\left(T_{k}^{\infty}\right)\right) \overline{a_{k}^{l+1}}
$$

Now the mean value theorem can be applied component-wise and yields for the $k$ th component

$$
\widehat{a}_{k}^{l+1}\left(\sigma_{k}\left(T_{k}^{l+1}\right)-\sigma_{k}\left(T_{k}^{\infty}\right)\right) \overline{\hat{a}_{k}^{l+1}}=\widehat{a}_{k}^{l+1} \sigma_{k}^{\prime}(\zeta) \overline{\hat{a}_{k}^{l+1}}\left(T_{k}^{l+1}-T_{k}^{\infty}\right)
$$

where $\zeta_{k}$ between $T_{k}^{l+1}$ and $T_{k}^{\infty}$, and $\sigma_{k}^{\prime}$ is by assumption non-positive. Hence, in matrix vector notation with $(\zeta)_{k}=\zeta_{k}$

$$
\left(\mathbf{M}_{\sigma}^{l+1}-\mathbf{M}_{\sigma}^{\infty}\right)\left(\widehat{\mathbf{a}}^{l+1} \circ \overline{\mathbf{a}^{l+1}}\right)=\mathbf{A}_{l+1} \mathbf{M}_{\sigma}^{\prime}(\zeta) \mathbf{A}_{l+1}^{\mathrm{H}} \mathbf{P}^{\top}\left(\mathbf{T}^{l+1}-\mathbf{T}^{\infty}\right)
$$

where $\mathbf{A}_{l}:=\operatorname{diag}\left(\hat{\mathbf{a}}^{l}\right)$ and the diagonal matrix $\mathbf{M}_{\sigma}^{\prime}(\zeta)$ has only non-positive elements, i.e., it is negative semi-definite. It follows

$$
\mathbf{L}\left(\mathbf{T}^{l+1}-\mathbf{T}^{\infty}\right)=h_{i} \frac{\omega^{2}}{2} \mathbf{P} \mathbf{M}_{\sigma}^{\infty}\left(\widehat{\mathbf{a}}^{l+1} \circ \overline{\widehat{\mathbf{a}}^{l+1}}-\widehat{\mathbf{a}}^{\infty} \circ \overline{\widehat{\mathbf{a}}^{\infty}}\right)+h_{i} \frac{\omega}{2} \mathbf{P} \operatorname{Im}\left(\widehat{\hat{\mathbf{j}}}_{\mathrm{s}} \circ \overline{\left(\widehat{\mathbf{a}}^{l+1}-\widehat{\mathbf{a}}^{\infty}\right)}\right)
$$

with $\mathbf{L}:=\mathbf{M}_{\rho c}+h_{i} \mathbf{N}-h_{i} \frac{\omega^{2}}{2} \mathbf{P} \mathbf{A}_{l+1} \mathbf{M}_{\sigma}^{\prime}(\zeta) \mathbf{A}_{l+1}^{\mathrm{H}} \mathbf{P}^{\top}$ whose inverse exists because it holds $\left\|\mathbf{L}^{-1}\right\| \leq\left\|\mathbf{M}_{\rho c}^{-1}\right\|$, since $\mathbf{M}_{\rho c}, \mathbf{N}$ are positive definite and $\mathbf{M}_{\sigma}^{\prime}(\zeta)$ is negative semi-definite. Multiplying (23) by the inverse of $\mathbf{L}$, taking the norm and exploiting the upper bound $\left\|\mathbf{M}_{\rho c}^{-1}\right\|$ yields

$$
\begin{aligned}
\left\|\mathbf{T}^{l+1}-\mathbf{T}^{\infty}\right\| \leq & \left\|\mathbf{M}_{\rho c}^{-1}\right\| \cdot\left[h_{i} \frac{\omega^{2}}{2}\left\|\mathbf{P M}_{\sigma}^{\infty}\left(\widehat{\mathbf{a}}^{l+1} \circ \overline{\mathbf{a}^{l+1}}-\widehat{\mathbf{a}}^{\infty} \circ \overline{\mathbf{a}^{\infty}}\right)\right\|\right. \\
& \left.+h_{i} \frac{\omega}{2}\left\|\mathbf{P} \operatorname{Im}\left(\widehat{\hat{\mathbf{j}}}_{\mathrm{s}} \circ \overline{\left(\widehat{\mathbf{a}}^{l+1}-\widehat{\mathbf{a}}^{\infty}\right)}\right)\right\|\right] .
\end{aligned}
$$

Now, we need estimates for (a) the linear term $\left\|\widehat{a}^{l+1}-\widehat{\mathbf{a}}^{\infty}\right\|$ and (b) the quadratic term $\left\|\widehat{\mathbf{a}}^{l+1} \circ \overline{\widehat{\mathbf{a}}^{l+1}}-\widehat{\mathbf{a}}^{\infty} \circ \overline{\widehat{\mathbf{a}}^{\infty}}\right\|$. 
(a) We start with the linear term: Consider (20) for the difference of exact solution and $(l+1)$ th iterate:

$$
\begin{aligned}
& j \omega \mathbf{M}_{\sigma}^{l} \widehat{\mathbf{a}}^{l+1}-j \omega \mathbf{M}_{\sigma}^{\infty} \widehat{\mathbf{a}}^{\infty}-\mathbf{X}\left(\widehat{\mathbf{a}}^{l+1}-\widehat{\mathbf{a}}^{\infty}\right)=0 \\
& \Leftrightarrow \quad \mathbf{Z}^{\infty}\left(\widehat{\mathbf{a}}^{l+1}-\widehat{\mathbf{a}}^{\infty}\right)+j \omega\left(\mathbf{M}_{\sigma}^{l}-\mathbf{M}_{\sigma}^{\infty}\right) \widehat{\mathbf{a}}^{l+1}=0 \\
& \Rightarrow \quad\left\|\widehat{\mathbf{a}}^{l+1}-\widehat{\mathbf{a}}^{\infty}\right\| \leq \omega\left\|\left(\mathbf{Z}^{\infty}\right)^{-1}\right\|\left\|\mathbf{M}_{\sigma}^{l}-\mathbf{M}_{\sigma}^{\infty}\right\|\left\|\widehat{\mathbf{a}}^{l+1}\right\| \\
& \leq \omega\left\|\left(\mathbf{Z}^{\infty}\right)^{-1}\right\|\left|\sigma_{\max }^{\prime}\right|\left\|\mathbf{T}^{l}-\mathbf{T}^{\infty}\right\|\left\|\left(\mathbf{Z}^{l}\right)^{-1}\right\|\left\|\widehat{\hat{\mathbf{j}}}_{\mathrm{s}}\right\| \\
& \leq C_{0}^{2}(\omega) \omega\left|\sigma_{\max }^{\prime}\right|\left\|\mathbf{T}^{l}-\mathbf{T}^{\infty}\right\|\left\|\widehat{\hat{\mathbf{j}}}_{\mathrm{s}}\right\| \\
& \leq c(\omega)\left\|\mathbf{T}^{l}-\mathbf{T}^{\infty}\right\| \text {, }
\end{aligned}
$$

where we have exploited the Lemma to obtain $c(\omega):=C_{0}^{2}(\omega) \omega\left|\sigma_{\max }^{\prime}\right|\left\|\widehat{\hat{\mathbf{j}}}_{\mathrm{s}}\right\|$.

(b) We now consider the quadratic term in (24)

$$
\begin{aligned}
\left\|\widehat{\mathbf{a}}^{l+1} \circ \overline{\mathbf{a}^{l+1}}-\widehat{\mathbf{a}}^{\infty} \circ \overline{\widehat{\mathbf{a}}^{\infty}}\right\| & =\left\|\left(\widehat{\mathbf{a}}^{l+1}-\widehat{\mathbf{a}}^{\infty}+\widehat{\mathbf{a}}^{\infty}\right) \circ \overline{\left(\widehat{\mathbf{a}}^{l+1}-\widehat{\mathbf{a}}^{\infty}+\widehat{\mathbf{a}}^{\infty}\right)}-\widehat{\mathbf{a}}^{\infty} \circ \overline{\widehat{\mathbf{a}}^{\infty}}\right\| \\
& \leq\left\|\left(\widehat{\mathbf{a}}^{l+1}-\widehat{\mathbf{a}}^{\infty}\right) \circ \overline{\left(\overline{\mathbf{a}}^{l+1}-\widehat{\mathbf{a}}^{\infty}\right)}\right\|+2\left\|\left(\widehat{\mathbf{a}}^{l+1}-\widehat{\mathbf{a}}^{\infty}\right) \circ \widehat{\mathbf{a}}^{\infty}\right\| .
\end{aligned}
$$

Because $\|a \circ b\| \leq\|a\|\|b\|$, this can be written as

$$
\begin{aligned}
\left\|\widehat{\mathbf{a}}^{l+1} \circ \overline{\widehat{\mathbf{a}}^{l+1}}-\widehat{\mathbf{a}}^{\infty} \circ \overline{\widehat{\mathbf{a}}^{\infty}}\right\| & \leq\left\|\widehat{\mathbf{a}}^{l+1}-\widehat{\mathbf{a}}^{\infty}\right\|^{2}+2\left\|\widehat{\mathbf{a}}^{l+1}-\widehat{\mathbf{a}}^{\infty}\right\|\left\|\widehat{\mathbf{a}}^{\infty}\right\| \\
& \leq c^{2}(\omega)\left\|\mathbf{T}^{l}-\mathbf{T}^{\infty}\right\|^{2}+2 c(\omega)\left\|\widehat{\mathbf{a}}^{\infty}\right\|\left\|\mathbf{T}^{l}-\mathbf{T}^{\infty}\right\|,
\end{aligned}
$$

where the Lemma gives us

$$
\left\|\widehat{\mathbf{a}}^{\infty}\right\|=\left\|\left(\mathbf{Z}^{\infty}\right)^{-1} \widehat{\mathbf{j}}_{\mathrm{s}}\right\| \leq C_{0}(\omega)\left\|\widehat{\hat{\mathbf{j}}}_{\mathrm{s}}\right\| .
$$

Using the estimates (28) and (29) in (24) gives

$$
\begin{aligned}
\left\|\mathbf{T}^{l+1}-\mathbf{T}^{\infty}\right\| \leq & h_{i} \frac{\omega}{2} c(\omega)\|\mathbf{P}\|\left\|\mathbf{M}_{\rho c}^{-1}\right\|\left\|\mathbf{T}^{l}-\mathbf{T}^{\infty}\right\|\left(\omega c(\omega)\left\|\mathbf{M}_{\sigma}^{\infty}\right\|\left\|\mathbf{T}^{l}-\mathbf{T}^{\infty}\right\|\right. \\
& \left.+2 \omega C_{0}(\omega)\left\|\widehat{\mathbf{j}}_{s}\right\|\left\|\mathbf{M}_{\sigma}^{\infty}\right\|+\left\|\widehat{\hat{\mathbf{j}}}_{\mathrm{s}}\right\|\right) .
\end{aligned}
$$

Now consider the asymptotic behavior of this for large $\omega$ and small $h_{i}$. It holds

$$
C_{0}(\omega)=\left\|\mathbf{X}^{-1}\right\|=\left\|\left(\omega^{2} \mathbf{M}_{\varepsilon}-\mathbf{C}^{\top} \mathbf{M}_{\nu} \mathbf{C}\right)^{-1}\right\| \sim \frac{1}{\omega^{2}}
$$

and

$$
c(\omega)=C_{0}^{2}(\omega) \omega\left|\sigma_{\max }^{\prime}\right|\left\|\widehat{\hat{\mathbf{j}}}_{\mathrm{s}}\right\| \sim \frac{1}{\omega^{3}} .
$$

Then, for fixed $h_{i}$ and $\omega$ large enough, it follows that

$$
\left\|\mathbf{T}^{l+1}-\mathbf{T}^{\infty}\right\| \leq h_{i} C(\omega)\left\|\mathbf{T}^{l}-\mathbf{T}^{\infty}\right\|,
$$

with some $C(\omega) \sim \frac{1}{\omega^{2}}$. 


\subsection{Generalization}

The frequency-transient model can be generalized in different ways. To enhance versatility, one can use a multi-frequency excitation of the EM subsystem. An application would be steel hardening of gears $[16,17]$, where two frequencies are necessary to obtain a homogeneous heating of the surface. Also to approximate periodic signals other than sinusoidal, multi-frequency excitation can be used. This also allows for a Harmonic Balance approach, which enables usage of a nonlinear permeability $\mu$.

\subsubsection{Time dependent phasor}

The model can be improved by weakening the assumptions made. In [4] it is suggested to consider the complex phasor not as constant within one time window. This means (10) becomes

$$
\mathbf{A}(t)=\frac{1}{2}\left(\hat{\mathbf{A}}_{c}(T) e^{j \omega t}+\overline{\hat{\mathbf{A}}}_{c}(T) e^{-j \omega t}\right)=\operatorname{Re}\left(\hat{\mathbf{A}}_{c}(T) e^{j \omega t}\right) .
$$

Thus the derivatives are

$$
\begin{aligned}
& \frac{\partial \mathbf{A}}{\partial t}=\operatorname{Re}\left(\frac{\partial \hat{\mathbf{A}}_{c}}{\partial t} \cdot e^{j \omega t}\right)+\operatorname{Re}\left(j \omega \hat{\mathbf{A}}_{c} \cdot e^{j \omega t}\right), \\
& \frac{\partial^{2} \mathbf{A}}{\partial t^{2}}=\operatorname{Re}\left(\frac{\partial^{2} \hat{\mathbf{A}}_{c}}{\partial t^{2}} \cdot e^{j \omega t}\right)+2 \operatorname{Re}\left(j \omega \frac{\partial \hat{\mathbf{A}}_{c}}{\partial t} \cdot e^{j \omega t}\right)-\operatorname{Re}\left(\omega^{2} \hat{\mathbf{A}}_{c} \cdot e^{j \omega t}\right),
\end{aligned}
$$

where $\hat{\mathbf{A}}_{c}:=\hat{\mathbf{A}}_{c}(T)$. Substituting (30)-(32) into (5) yields

$$
\nabla \times\left(\mu^{-1} \nabla \times \hat{\mathbf{A}}_{c}\right)+\varepsilon\left(\frac{\partial^{2} \hat{\mathbf{A}}_{c}}{\partial t^{2}}+2 j \omega \frac{\partial \hat{\mathbf{A}}_{c}}{\partial t}-\omega^{2} \hat{\mathbf{A}}_{c}\right)+\sigma(T)\left(\frac{\partial \hat{\mathbf{A}}_{c}}{\partial t}+j \omega \hat{\mathbf{A}}_{c}\right)=\hat{\mathbf{J}}_{\mathrm{src}}
$$

which is now a second order PDE with partial derivatives with respect to time as well. Note that in [4] magnetoquasistatic formulation is used, so there the modification yields a first order system. However, since now both parts (EM and heat) have to be time integrated, the co-simulation of these can be called dynamic iteration [3].

Due to the modification (12) changes to

$$
\begin{aligned}
\tilde{Q}(T)= & \frac{\omega^{2}}{2} \sigma(T)\left\|\hat{\mathbf{A}}_{c}\right\|_{c}^{2}+\frac{\omega}{2}\left[2 \sigma \operatorname{Im}\left(\frac{\partial \hat{\mathbf{A}}_{c}}{\partial t} \cdot \overline{\hat{\mathbf{A}}_{c}}\right)+\operatorname{Im}\left(\hat{\mathbf{J}}_{\mathrm{src}} \cdot \overline{\hat{\mathbf{A}}_{c}}\right)\right] \\
& +\frac{1}{2}\left[\left\|\frac{\partial \hat{\mathbf{A}}_{c}}{\partial t}\right\|_{c}^{2}+\operatorname{Re}\left(\overline{\hat{\mathbf{J}}_{\mathrm{src}}} \cdot \frac{\partial \hat{\mathbf{A}}_{c}}{\partial t}\right)\right] .
\end{aligned}
$$

The convergence analysis can be extended accordingly.

\subsubsection{MPDAE approach}

The Multirate Partial Differential Algebraic Equations (MPDAE) approach in [18] offers another type of generalization. It yields a fully time-domain model that introduces two time scales according to a fast and a slow component in the solution. For circuit simulation this can be exploited to efficiently determine envelope simulation in case of amplitude modulation. The method however also works for frequency modulation problems. For the coupled electromagnetics-heat problem the slow time scale comes from the heat equation, the fast time scale comes from the periodic source in the electromagnetic problem. 


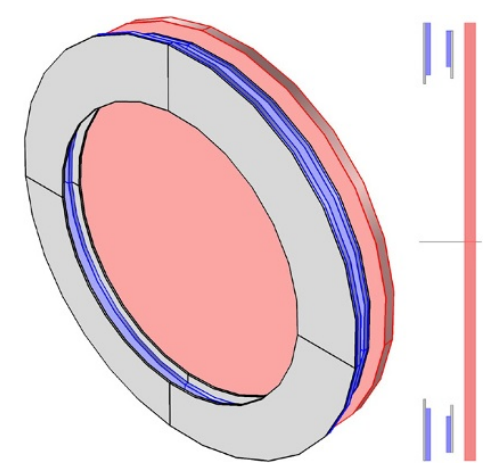

(a) $3 \mathrm{~d}$ view and $2 \mathrm{~d}$ cut view on geometry. From left to right: ferrite (gray), primary coil (blue), air, secondary coil (blue), ferrite (gray), air, steel slice (red). The left coil represents the charging station and the right coil the coil behind the number plate in the car.

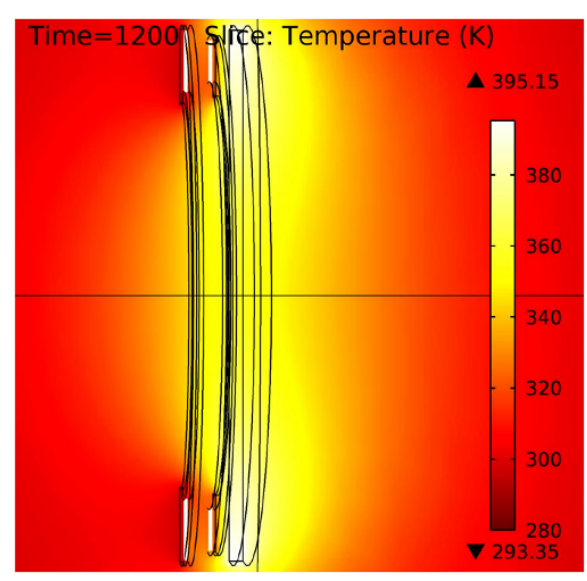

(b) Temperature distribution after a simulation time of $20 \mathrm{~min}$. The maximum temperature is $395.15 \mathrm{~K}$. This is the cross section as shown in (a).

Figure 4 Model for an inductive charging system for electric vehicles (Comsol).

\section{Frequency-transient co-simulation example}

In this section the frequency-transient model is applied to an inductive charging system for electric vehicles. The charging system is modeled in a $2 \mathrm{~d}$-axisymmetric way by a primary coil for the station and a secondary coil as receiver in the car. Both coils include ferrite. Additionally there is a steel slice to model the part of the car body behind the secondary coil. The electric conductivity of this steel slice depends on the temperature, i.e., the system is mutually coupled. The geometry and simulation results are shown in Figure 4. For more details of the set up we refer to [2].

A constant conductivity would have lead to a single way coupled system and thus the magnetic vector potential would by a constant vector phasor. Models with a constant conductivity (according to the initial temperature) will systematically underestimate the heating. In this case the obtained maximum temperature would be $7 \mathrm{~K}$ below the corresponding solution of the mutually coupled problem, [2].

For mutually coupled problems the frequency-transient model has proved to be very efficient. This is expected, since the main part of the computational effort - the time integration of the EM subsystem - is avoided. In this numerical example $\omega$ was chosen to be $2 \cdot \pi \cdot 10 \mathrm{kHz}$. In fact the proposed co-simulation algorithm reached the end time of $t_{\text {end }}=20$ min by using only 17 time steps with $n \leq 14$ iterations (on average $\bar{n} \approx 5.4$ iterations). This coarse time grid sufficiently resolves the dynamics of the heat equation. For comparison, in a naive monolithic simulation with 10 time steps per period 120 million time steps would be necessary to resolve the dynamics of the EM subsystems. This underlines the computational gain.

\section{Conclusions}

A frequency-transient model tailored for heat-electromagnetic problems was derived. The time step size of the coupled system is determined by the heat subsystem only. The con- 
vergence analysis is presented in detail. Convergence for high frequencies is guaranteed. The error bound for the iteration decreases quadratically with higher frequencies. This result also applies to approaches by Driesen and Hameyer [4] and similar implementations in Comsol [19]. Thus the approach fits perfectly for applications where inductive heating either appears as losses or is intended.

\section{Competing interests}

The authors declare that they have no competing interests.

\section{Authors' contributions}

All authors contributed to this paper as a whole. However, special merits go to DK and MR for sharing their experiences from industry, which led to the numerical example and introduction; to SS and MG for the work on the model; to CK, JtM and MK for their contribution to the analysis. All authors read and approved the final manuscript.

\section{Author details}

${ }^{1}$ Fachbereich Elektrotechnik und Informatik, Hochschule Bochum, Bochum, 44801, Germany. ${ }^{2}$ Lehrstuhl für Angewandte Mathematik/Numerische Analysis, Bergische Universität Wuppertal, Wuppertal, 42119, Germany. ${ }^{3}$ Technology Development and Qualification - Simulation, Leopold Kostal GmbH \& Co. KG, Lüdenscheid, 58513, Germany. ${ }^{4}$ Graduate School of Computational Engineering, Technische Universität Darmstadt, Darmstadt, 64293, Germany. ${ }^{5}$ Department of Mathematics \& Computer Science, TU Eindhoven, PostBox 513, MB Eindhoven, 5600, The Netherlands.

\section{Acknowledgements}

This work is supported by the German BMBF in the context of the SOFA project (grant number 03MS648E). The sixth author is supported by the 'Excellence Initiative' of the German Federal and State Governments and the Graduate School of Computational Engineering at Technische Universität Darmstadt.

Received: 23 April 2013 Accepted: 24 March 2014 Published: 3 June 2014

\section{References}

1. Schneider D: Wireless power at a distance is still far away [Electrons unplugged]. IEEE Spectr 2010, 47(5):34-39.

2. Kaufmann C, Günther M, Klagges D, Richwin M, Schöps S, ter Maten EJW: Coupled heat-electromagnetic simulation of inductive charging stations for electric vehicles. In Progress in Industrial Mathematics at ECMI 2012. Berlin: Springer; 2014. [Mathematics in Industry.]

3. Bartel A, Brunk M, Günther M, Schöps S: Dynamic iteration for coupled problems of electric circuits and distributed devices. SIAM J Sci Comput 2013, 35(2):B315-B335.

4. Driesen J, Hameyer K: The simulation of magnetic problems with combined fast and slow dynamics using a transient time-harmonic method. Eur Phys J Appl Phys 2001, 14:165-169.

5. Schöps S, De Gersem H, Weiland T: Winding functions in transient magnetoquasistatic field-circuit coupled simulations. Compel 2013, 32(6):2063-2083.

6. Haus HA, Melcher JR: Electromagnetic Fields and Energy. New York: Prentice Hall; 1989 [http://web.mit.edu/6.013_book/www/]

7. Clemens M, Gjonaj E, Pinder P, Weiland T: Numerical simulation of coupled transient thermal and electromagnetic fields with the finite integration method. IEEE Trans Magn 2001, 36(4):1448-1452.

8. Clemens M, Gjonaj E, Pinder P, Weiland T: Self-consistent simulations of transient heating effects in electrical devices using the finite integration technique. IEEE Trans Magn 2001, 37(5):3375-3379.

9. Weiland T: Time domain electromagnetic field computation with finite difference methods. Int J Numer Model 1996, 9(4):295-319.

10. Bossavit A: Computational Electromagnetism: Variational Formulations, Complementarity, Edge Elements. San Diego: Academic Press; 1998 [http://tut.fi/ bossavit/\#Books]

11. Hiptmair R, Ostrowski J, Quast R: Modelling and simulation of induction heating. Technical Report 149, SFB 382, University of Tübingen; 2000.

12. Alotto P, Freschi F, Repetto M: Multiphysics problems via the cell method: the role of Tonti diagrams. IEEE Trans Magn 2010, 46(8):2959-2962.

13. Hahne $P$, Weiland $T: 3 D$ eddy current computation in the frequency domain regarding the displacement current. IEEE Trans Magn 1992, 28(2):1801-1804.

14. Clemens $M$, Weiland T: Numerical algorithms for the FDiTD and FDFD simulation of slowly varying electromagnetic fields. Int J Numer Model Special Issue 'Finite Difference Time Domain and Frequency Domain Methods', Invited Paper 1999, 12(1/2):3-22.

15. Chen Q, Schoenmaker W, Chen G, Jiang L, Wong N: A numerically efficient formulation for time-domain electromagnetic-semiconductor co-simulation for fast-transient systems. IEEE TCAD 2013, 32(5):802-806

16. Rudnev V: Induction hardening of gears and critical components. Part I. Gear Techno/ 2008, 2008:58-63.

17. Rudnev V: Induction hardening of gears and critical components. Part II. Gear Techno/ 2008, 2008:47-53.

18. Brachtendorf HG, Welsch G, Laur R, Bunse-Gerstner A: Numerical steady state analysis of electronic circuits driven by multi-tone signals. Electr Eng 1996, 79:103-112 [doi:10.1007/BF01232919].

19. COMSOL Multiphysics: Command reference; 2007 [wWw.comsol.com]

doi:10.1186/2190-5983-4-1

Cite this article as: Kaufmann et al.: Efficient frequency-transient co-simulation of coupled heat-electromagnetic problems. Journal of Mathematics in Industry 2014 4:1 